\title{
Partial Discharges Behaviors of Internal Void in the Oil-Paper Insulation and its Effect on the Material
}

\author{
Weizheng Zhang ${ }^{\mathrm{a}}$, Zhimin Li, Guojian Ji, Jumei Guo, Yongbo Yang \\ Zhengzhou Power Supply Company of the State Grid Henan Electric Power Company, \\ Zhengzhou, ${ }^{2} x j t u z w z @ 163 . c o m$
}

Keywords: partial discharge, oil-impregnated paper, thermally aging, pulse sequence analysis

\begin{abstract}
Because of the complexity of transformers construction, internal voids in the insulation seems inevitable when fabricating, which would cause partial discharge (PD). Therefore, it is of great significance to monitor the PD to study in which condition the PD will make the insulation degradation and whether there have diversities among different PDs. In this paper, the oil-immersed paper samples are artificially thermally aged to obtain specimen with different aging states, and the continuous test on oil impregnated paper with internal void defect are developed and the phase resolved partial discharge (PRPD) pattern of PD signals during the electrical aging process are measured. The experimental results show that the PD behaviors in internal void of the oil-paper insulation have couples of transient processes and the mechanisms behind are quite complicated. The pulse sequence analysis results validate that thermally aging condition of paper have great impact on PD characteristics, which show that the pulse number increase and time between consequence discharge get closer. The study would be helpful to possibly determine the discharge type and condition assessment of power transformer with the PD monitor.
\end{abstract}

\section{Introduction}

The oil-impregnated paper composite insulation has been widely used over a century, because of its good performance in many high-voltage equipment such as large transformers, transformers, bushings, etc. , and occupies an important position in the power system. Partial discharge and insulating materials is closely related to aging and breakdown, thereby affecting the life of the high-voltage electrical equipment, so the partial discharge detection and recognition is one of the effective tools to ensure the safe and stable operation of large power equipment ${ }^{[1]}$. Important incentive for both partial discharge of oil-paper insulation system degradation, but also one of the important signs of the development process and the impact of research on partial discharge insulation materials contribute to a better understanding of the mechanism of partial discharge and its relation to the aging of the insulation material, and then there is may provide a basis for understanding the phenomenon obtained based on the measured electrical equipment inside the discharge characteristics and the insulation state ${ }^{[2]}$.

However, due to the insulating effect of electrical equipment complex work environment ${ }^{[1,2]}$, oil-paper insulation by electrical, thermal, mechanical and environmental stresses during operation gradually age, and physical and chemical properties of the medium gradually change, what impacting its partial discharge features and performance, thereby strengthening the partial discharge. At present, domestic and cross-impact on oil-paper insulation between different partial discharge its aging state relations and partial discharge assessment of the dangers of oil-paper insulation has not been able to solve, which is restricting the partial discharge measurement in the effectiveness of on-site implementation bottlenecks.

This paper prepared different laboratory artificial heat aging of oil-paper insulation aging as research object, by means of PD meter, digital oscilloscopes and scanning electron microscopy, to study the development of oil-paper insulation inside the air-gap discharge, the discharge phase characterization methods and discharge of insulating materials deterioration affected. 


\section{Experimental Design}

Experimental platform. Laboratory set up in line with IEC60270 standard partial discharge test platform, which shows in Fig.1. The digital oscilloscope and partial discharge detector record pulse waveform and its statistical characteristics of partial discharge signals simultaneously. Test AC power supply with no PD test transformer, rated voltage $100 \mathrm{kV}$, rated power $10 \mathrm{kVA}$, at $100 \mathrm{kV}$ partial discharge under less than 5pC. During the test, PD measurement system using Italy TechImp PDBase system which can record the PD Phase Resolved Partial Discharge (PRPD) spectra, in order to distinguish between the various spectra of PD evolution. Because PD meter output is an analog signal, which can not give quantitative information, so we regard it as qualitative observations in this to determine whether the discharge, the discharge intensity, frequency, size and so on. while quantitative information, such as discharge pulse waveform sequence and a single pulse, a digital storage oscilloscope measured through a non-inductive resistance. Using digital oscilloscope Tektronix 4034, its maximum bandwidth of up to $500 \mathrm{MHz}$, the maximum sampling frequency of $2.5 \mathrm{GSa} / \mathrm{s}$.

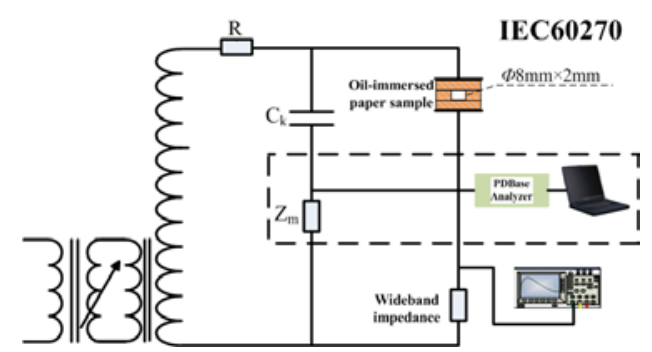

Fig.1 partial discharge test route map

$\mathrm{R}$----- protection resistor $C_{\mathrm{k}}$----- coupling capacitor $Z_{\mathrm{m}}$-----measured impedance

Oil-paper prepared by different degrees of aging test. In order to make the insulating sheet can be sufficiently immersed in oil under vacuum, the housing is provided with a vacuum oven fuel inlet and fill valve. When required vacuum oven, the fuel injection valve is closed, opens the vacuum pump and the gas vent valve; When insulating paper vacuum immersion oil, grease valve is opened and the vacuum pump is turned on at the same time pay attention to controlling the flow rate of the transformer oil so that it slowly into the vacuum oven interior to reduce bubbles, fully impregnated paper insulation. In addition, the internal model to simulate the air gap between the discharge is not fully impregnated paper layers to form a bubble phenomenon, a "sandwich" structure, namely the middle with $2 \mathrm{~mm}$ cardboard insulation, cut out the center of an $8 \mathrm{~mm}$ diameter cylindrical, up and down the cover $0.13 \mathrm{~mm}$ the formation of the air gap insulation paper, and then cast resin molding. Aging of oil-paper sample in a vacuum oven vacuum impregnation, and then subjected at $140{ }^{\circ} \mathrm{C} 50$ hours, 330 hours and 800 hours heat aging, to prepare the different stages of aging test.

Experiment and analysis methods. Studies have shown that most of the discharge situation is that the partial discharge intensity gradually increased, so that the material continuously deteriorates partial discharge at this time is a dynamic process of development, what is possible to understand the basis of the measurement results of properties of the material, and the life of the material. In this paper, partial discharge test platform for all types of oil-paper test were prepared ladder boost, recording PRPD spectrum evolution of each voltage, combined with changes in oil-paper paper SEM observation sample surface.

Although partial discharge event is random, but the decision phase of PD pulses generated by more than occurred in front of the discharge, but by the previous discharge phase - the combined effect of the magnitude that the magnitude of the time difference with PD pulses is mutual parameters associated. In this paper, the use of pulse sequence analysis of partial discharge signal analysis, in order to analyze the different development stages of PD discharge characteristic quantities which considers the two discharges $\Delta u$ spectrum "memory effect" ${ }^{[3]}$, estimated two 
consecutive PD produced the desired discharge pulse voltage difference frequency of occurrence. Use $\Delta \mathrm{u} / \Delta \mathrm{t}$ or $\Delta \mathrm{u} / \Delta \phi$ represents a continuous spectrum of PD pulses required potential gradient.

\section{Results and discussion}

Physical and chemical analysis of the aging oil-paper. Prolonged overheating will accelerate the deterioration of oil-paper insulation, paper fibers usually presents cleavage, long-chain structure broken into short-chain structure, formation of aldehydes dissolved in oil; impurities in the oil, acid value, dielectric loss and other indicators significantly higher. For four different age levels of physical and chemical analysis of oil-paper test results are shown in Table 1. The results show that the high temperature thermal aging effects on the physical and chemical properties of oil-paper insulation paper test significantly. Oleic acid value, dielectric loss, resistivity and furfural as a significant increase in the degree of aging rise significantly decreased the degree of polymerization. 800 hours aging test paper reduced the degree of polymerization 311, close to the end of oil-paper insulation transformer design life period.

Table 1 different aging degree of physical and chemical analysis of the test results oil-paper

\begin{tabular}{|c|c|c|c|c|c|}
\hline Degree of aging & $\begin{array}{c}\text { Oleic acid } \\
\text { value(mgKOH/g) }\end{array}$ & Oil $\tan \delta(\%)$ & $\begin{array}{c}\text { Oil } \\
\text { Resistivity }(\Omega . m)\end{array}$ & $\begin{array}{c}\text { Oil } \\
\text { furfural(mg/L) }\end{array}$ & $\begin{array}{c}\text { Paper } \\
\text { polymerization } \\
\text { degree }\end{array}$ \\
\hline New & 0.007 & 0.03 & $3.20 \mathrm{E}+10$ & 0.01 & 928 \\
\hline $50 \mathrm{~h}$ & 0.008 & 0.04 & $3.00 \mathrm{E}+10$ & 0.745 & 733 \\
\hline $330 \mathrm{~h}$ & 0.015 & 0.15 & $1.70 \mathrm{E}+10$ & 2.853 & 420 \\
\hline $800 \mathrm{~h}$ & 0.049 & 2.56 & $1.50 \mathrm{E}+10$ & 8.04 & 311 \\
\hline
\end{tabular}

PRPD evolution whit internal gap PD. Our research shows that oil-paper insulation inside the air-gap discharge process affected by oil-paper paper surface conditions, the impact of the size of the air gap volume and other factors. The discharge would have evolved a variety of forms, with a very complex the discharge mechanism. PRPD spectral evolution under $5.5 \mathrm{kV}$ of aging 50 hours inside the air-gap discharge test AC voltage is shown in Fig.2 . Fig.2 (a), (b), (c) and (d) respectively load applied voltage $1 \mathrm{~min}, 5 \mathrm{~min}, 7 \mathrm{~min}$ and $12 \mathrm{~min}$ (before the breakdown). For oil-paper insulation inside the air gap in the initial discharge showing a large amount of discharge, number of pulses of the characteristics of the gas molecules react violently with each other in this process, large quantities consumed. After five minutes discharge capacity and the number of pulses are significantly reduced, PRPD spectrum exhibits the two "hump-shaped". This process does not last long, which turn into "rabbit ear". Final discharge capacity decreases slowly, maintained to the previous breakdown. It should be noted, changes in PD mode caused by a variety of factors, such as changes in the gaseous state, the deterioration of the product, the paper surface conditions, the effect of surface charge generated front discharge ${ }^{[4]}$, but so far the spectrum paradigm shift mechanisms are not yet fully understood.
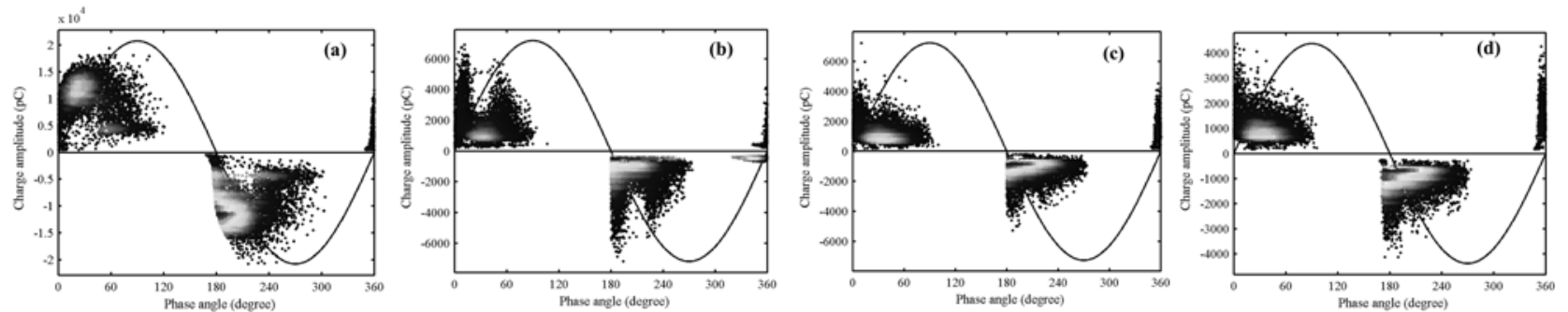

Fig.2 Internal air gap discharge PRPD spectra evolution with 50 hours aging test under 5.5kV AC

PD pulse sequence analysis of different degrees aging test. Pulse sequence analysis proposed by the German Professor R.Patsch and M.Hoof firstly ${ }^{[5]}$, inspired by the statistics office put the required applied voltage pulse to an understanding of the difference between the partial discharge 
behavior characteristic form $\Delta \mathrm{u}$ spectrum. Compared with PRPD traditional spectrum, pulse sequence analysis method considers the impact on front-discharge follow-up discharge. $\Delta t$ spectrum counted twice in the discharge time difference between the different stages of development to distinguish discharge. Different aging stages of oil-paper insulation partial discharge test spectra and $\Delta t$ and $\Delta \mathrm{u}$ spectrum shown in Fig.3. It can be seen that with the aging of the test deepen, $\Delta t$ spectra showing the trend to move to the left, that is a serious test of aging interval between two discharges significantly smaller. The new oil-paper test time difference between two successive discharges is about 3-10ms, while aging 800 hours of concentrated within 1-8ms range.
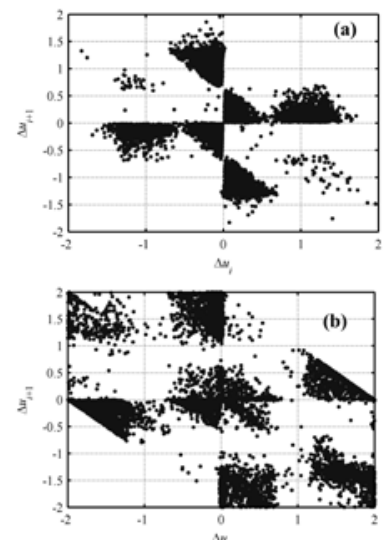

Fig.3 different levels of aging oil-paper insulation test PD pulse sequence analysis
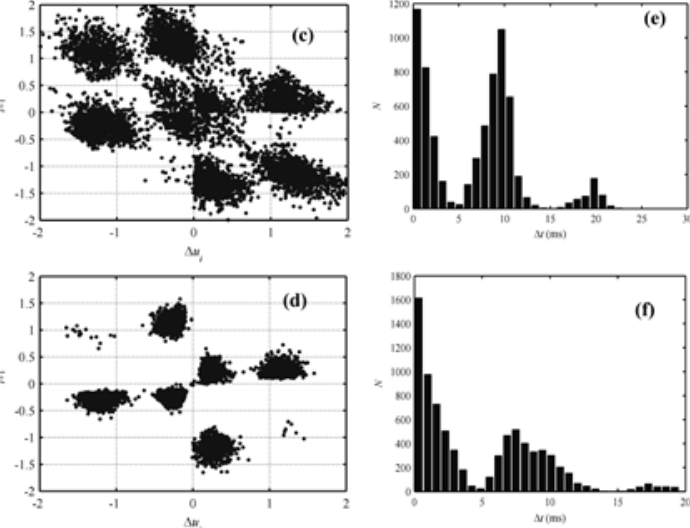
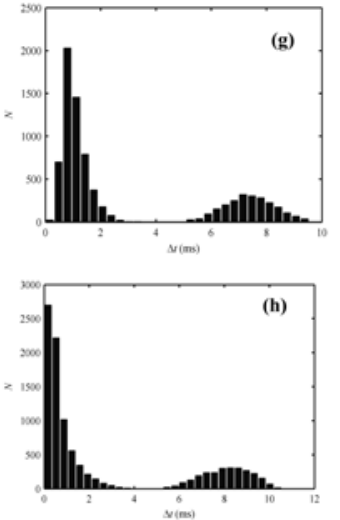

(a) - (d) are new, aging 50h, 330h, 800h sample of $\Delta \mathrm{u}$ map; (e) - (h) for the corresponding $\Delta \mathrm{t}$ map

SEM images of specimen surface. Fig.4 shows different aging of oil-paper SEM of the sample surface. The Fig.scale is 100 times. As can be seen from the figure, the new paper into the surface of the fiber through the vacuum impregnation uniform structure, compact. After a long period after thermal aging (Fig.4 (c) and (d)), the paper fibers significantly more brittle fracture , and the surface clutter intertwined. In summary, the SEM image shows that the cardboard, paperboard with increased aging, degradation of cellulose fibers by the effect of factors and destruction, its length becomes shorter, smaller thickness, the surface rougher, more sparse structure, gaps between fibers gradually increase interaction large, between fibers gradually weakened.

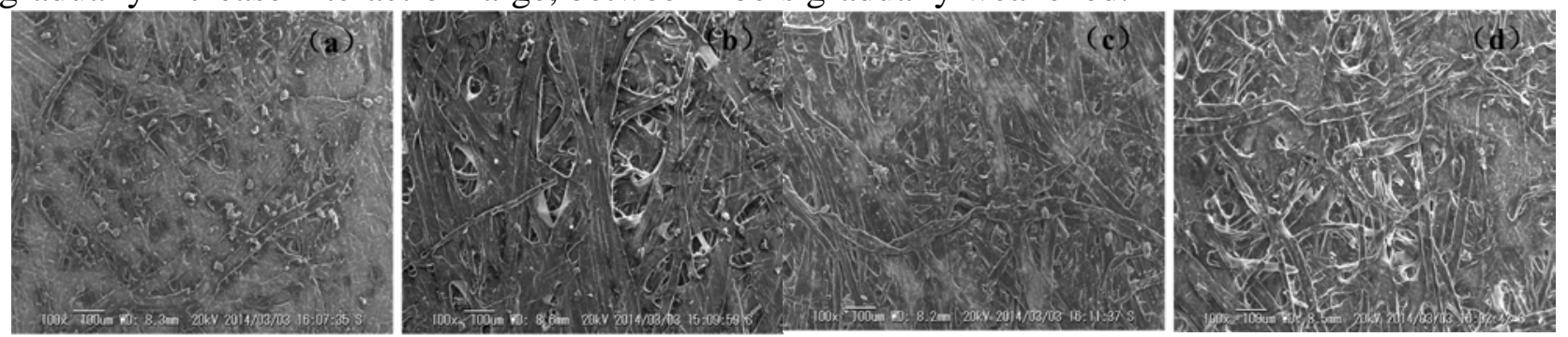

Fig.4 SEM images of Samples withstand strong discharges after direct breakdown
(a)new
(b) $50 \mathrm{~h}$
(c) $330 \mathrm{~h}$
(d) $800 \mathrm{~h}$

\section{Conclusion}

This paper, by means of partial discharge detector and SEM, study the development of local properties and thermal aging of oil-paper insulation of different sample of the discharge of the effects of aging on the insulating paper. Study confirmed the prolonged overheating will accelerate the deterioration of oil-paper insulation, paper fiber cracking performance, long-chain structure broken into short-chain structure, formation of aldehydes dissolved in oil; impurities in the oil, acid value, dielectric loss and other indicators increased significantly . Development of oil-paper insulation inside the air-gap discharge discharge spectrum there are several changes, changes in playback mode Council by a variety of factors, such as changes in the gaseous state, the deterioration of the product, the paper surface conditions, the impact of the previous generation of 
surface charge and discharge. After partial discharge pulse sequence analysis it concluded that the impact of aging for PD performed that the number of pulses increased and the time difference between successive discharges significantly reduced.

Oil-paper surface of the sample by SEM different aging degree scan results show that with an increase in aging cardboard, cellulosic fibers to degradation factors and destruction, its length becomes shorter, roughness becomes smaller, more rough the surface, the structure is more sparse, and gradually increase the gap between the fibers, the interaction between the fibers are waning.

Oil-paper insulation inside the air-gap discharge process by oil-paper paper surface conditions, such as multiple factors influence the size of the air gap volume, discharge would have evolved a variety of forms. The discharge mechanism is very complex and requires further study.

\section{References}

[1] Gao Zhenguo,Han Gui,Gui Jingfan. Power transformer fault diagnosis dendritic discharge. Transformer, 1994, 31(4):17 20

[2] M. Hoof and R. Patsch, "Analyzing partial discharge pulse sequences-A new approach to investigate degradation phenomena”, 1994 IEEE International Symposium on Electrical Insulation, Pittsburgh, PA USA, June 5-8, 1994, pp. 327-331

[3] R. Patsch, F. Berton, "Pulse Sequence Analysis - a diagnostic tool based on the physics behind partial discharges”, J. Phys. D: Appl. Phys., vol. 35, No. 1, pp.25-32, 2002.

[4] C.S. Kim, T. Kondo and T. Mizutani, “Change in PD Pattern with Aging”, IEEE Trans. Diele. Electr. Insul., pp. 13-18, 11(1), Feb. 2004.

[5] A. Contin and H. Al-Marzouqi, "Searching for partial discharge patterns for the identification of defects of insulation systems in AC rotating machines”, IEEE Electrical Insulation Conference, Montreal, QC, Canada, June 2009. 